\title{
ANALYSIS OF SMS FEEDBACK AND ONLINE FEEDBACK USING SENTIMENT-ANALYSIS FOR ASSESSMENT OF TEACHING
}

\author{
Santosh S Patil ${ }^{1}$, Uttej Seemakurty ${ }^{2}$, Valarmathi $\mathbf{B}^{3}$ \\ ${ }^{1}$ Asst. Prof, School of Engineering, CUK, Kalaburagi, Karnataka, India \\ ${ }^{2}$ Software Engineer, Accenture Service Pvt Ltd, Chennai, Tamilnadu, India \\ ${ }^{3}$ Asst. Prof, SITE, VIT Vellore, Tamilnadu, India
}

\begin{abstract}
In this, the system will collect SMS's and online Feedback from students in free text format. To get instance response of lecturer feedback by analyzing free text messages using sentiment analysis. It is very low cost and plat form independent. It will show you three different outputs, i.e. Good, Bad and Average/Neutral. The result will be shown in graphical format.
\end{abstract}

Key Words: Sentiment analysis, online feed-back.

\section{INTRODUCTION}

In this we introducing the feedback from student by collecting through SMS's and online using free text for assessment of teaching. In traditional method, the assessment of the teacher is done by students in the format of Multiple Choice Question (MCQ) or polling using SMS facility. In the MCQ, the feedback may be a single-alphabet (A or B or C or D) and in the case of polling, either we can select Yes (Y) or No (N) to give feedback about the teacher. In the proposed system, the assessment of the teacher is done by students in the form of free text. These SMS's are collected by the System. Then it will analyze the SMS's and gives the feed-back of teacher by using Sentiment Analysis. The system will have lecturer and student login pages. In student login, there is an option to give feedback with many categories (teaching, behaviour, communication, knowledge, interaction) of a teacher. The system will check the spelling and grammar errors for the free text given by the student and it will highlight the errors, for changing to correct one. In Lecturer login, we will show the feedback in graphical representation for all categories and overall presentation also.

\section{LITERATURE SURVEY}

Zhang et al, proposed an important system application in text mining. Sentiment detection is becoming more and more important, due to the expanding volume of available online information, such as micro-blogging messages and review comments. As a result, a lot of sentiment detection methods have been developed, among which machine learning methods have received a lot of interest.

Michael Hagenau et al proposed a system in which it examines stock price. Effects can be automatically predicted by analyzing unstructured-textual information in financial news. The main contribution is the usage of expressive features to represent text feedback as part of our word selection process. The methodology can be transferred to any other application area providing textual information and corresponding effective data.

Pete Rotella and Sunita Chulani has proposed a system in which it conducts a Customer Satisfaction survey (CSAT) each year to gauge customer sentiment regarding products, technical support, technical services, order fulfilment, and a number of other aspects of the company's business using free text. As a result, the company can make changes according to the survey and user's requirements.

Karma soft gave a detail description for SPELL-CHECK for free text in ASP.NET. It is using Microsoft office word's spell- checking method. The text data will save in word document and checks the spell. It will give you an option to change the mistakes, if any.

Llia Smirnov gave the varieties of stemming algorithms. Stemming is to change the words to its root word. For example Teaching is changed to Teach.

Willett Peter gave briefly explained about Porter Stemming Algorithm. He explained how the algorithm works before and after. He tells about efficiency and it's important also.

Steven Abney gave how to do a POS-Tagging and parsing simultaneously. POS-Tagging is to find the parts of speech for a sentence includes parsing. Parsing is used in Natural language.

By using this SMS's and online feedback, the assessment of the teacher will be more faster and easy. This method will be more useful in educational institutions for the teaching evaluation. By using this, we can get the instant response from the students. The students will also interestingly participate in this survey. For this method, we need an internet enable computer and mobile-phone. 


\section{PROPOSED SYSTEM}

Feedback will be taken from 2 ways. First is through student login. Second is through SMS. The database will maintain the login detail of student and staff. The data that entered in student login page will save in database along with SMSs that are received for a particular teacher. We will merge those data in a staff id. Then we will analyze the data with sentiment analysis method for result. The result should be shown in lecturer login in the form of chats for better understanding purpose. The comments and messages that are received for lecturer also shown in their login.

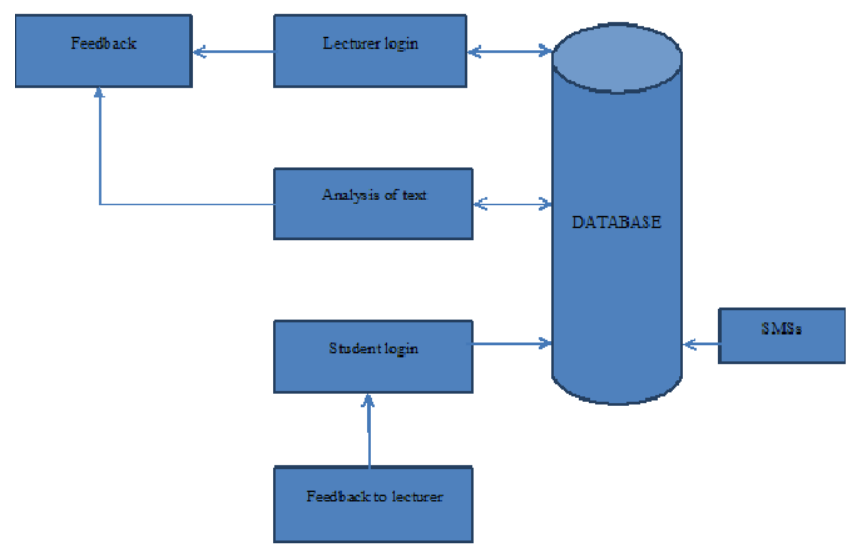

Fig -1: System Architecture

In this system, we have 10 modules. The introduction of each module is given below.

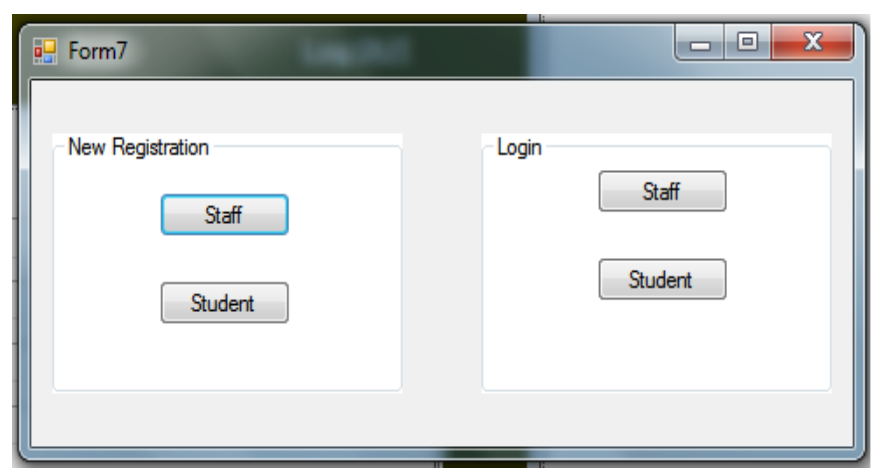

Fig -2: Login Screen

Student Login: In this, Student can give feedback to many categories (teaching, behaviour, communication, knowledge, interaction) of a particular lecturer for respective class. The student should register first in StudentRegistration page.

Lecturer login: In this, Lecturer can see the messages of the student and feedback of a particular class with graph format for overall feed-back and another graph for individual categories. The lecturer should also register first in StaffLogin page.

Collection of SMSs and Messages: First step is to collect messages from the students in a particular subject through SMSs and from student login. Next step is to create database for the collected messages. Database contains number of messages received from the students, received message, date and time.

Corrected model: In this, there is spell checker. We can correct the spelling mistakes in each document, if any. It will suggest some words from dictionary. We select from the list for changing it.

Grammar Correction: In this, we correct the grammar of a sentence in each document, if any. It will show the correct sentence options for changing.

Stop Word Removal: It will remove the stop-words like to, $a$, an, not, the, etc. It will help for easy analyzing purpose in sentiment analysis module.

Stemming: It is process of mapping of all the alternatives to the ROOT word. It will identify the tense of the word. For example, the root word "go" has the following variations go, went, gone.

Parsing: It will parse the data and tag the parts-of speech for every word.

Sentiment Analyzer: This is very important module. The system will analyze each and every message in relevant group individually using sentiment analysis. It gives each and every person feedback as positive or negative or average about the lecture in graph format.

\section{RESULT}

The user can give the feedback in different forms. If the user types wrong spelling or incorrect grammar it will show immediately to user for changes. So by this correction the analysis of text will be easier. We can show the result immediately to the user for their feedback. The result will be in three forms, i.e. good, bad, and average.

\begin{tabular}{|c|c|}
\hline WORDS in sentence & RESULT \\
\hline Good & GOOD \\
\hline Not good & AVERAGE \\
\hline Not bad & GOOD \\
\hline Bad & BAD \\
\hline Other data/irrelevant data & AVERAGE \\
\hline Empty space & AVERAGE \\
\hline
\end{tabular}

The result of this methodology is a graphical representation in lecturer login page. We are showing different graphs for different categories and a graph for overall status from SMS and online feedback. We will show the messages that are collected from SMS and suggestions from student login in lecturer login page. 


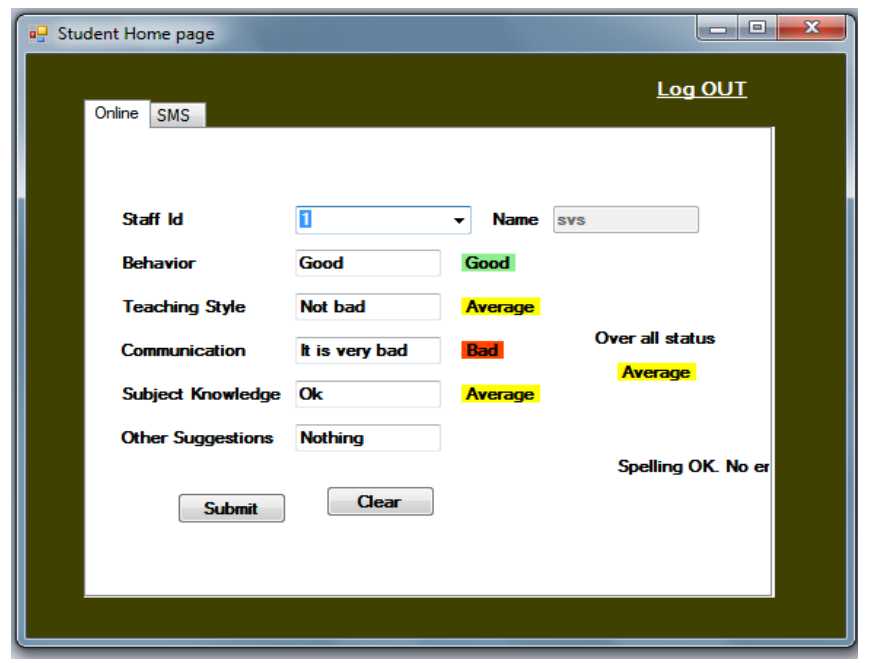

Fig -3: Student Home page

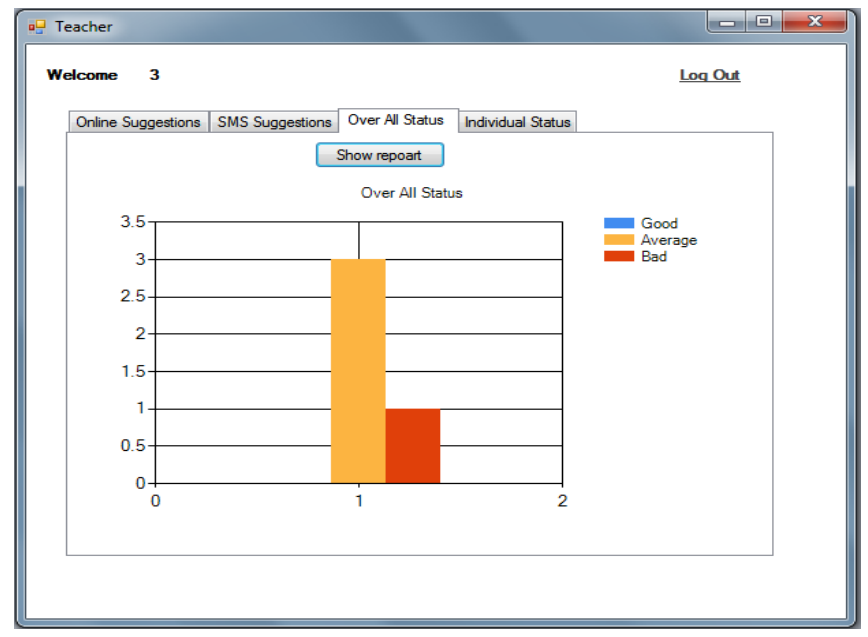

Fig -4: Feedback result analysis

\section{CONCLUSIONS}

By this methodology we can give feed-back to lecturer. It will correct spelling and grammar in text entered by the user. We will show the result in graphical format for better understanding to lecturer. This is useful for improvement of his/her performance. In future this can be developed as ANDROID-APP for simple and fast response. We can use this method for various other categories. This is very low cost and platform independent.

\section{REFERENCES}

[1]. Chee Kian Leong et al, "Mining sentiments in SMS texts for teaching evaluation", Expert Systems with Applications, pp. 2584-2590, 2012

[2]. Dan Zhang et al, "Sentiment Detection with Auxiliary Data", Conference of Department of Computer Science, Purdue University, US, 2012

[3]. James Lee, "Beginning-Perl", Apress, www.perl.org/books/beginning-perl

[4]. Michael Hagenau et al, "Automated NEWS reading: Stock Price Predictions Based on Financial News", $45^{\text {th }}$ Hawaii International Conference on System Sciences, 2012
[5]. Pete Rotella and Sunita Chulani, "Analysis of Customer Satisfaction Survey Data", Cisco Systems Conference, USA, 2012

[6]. Karma soft, "Spell-Checker for ASP.NET using Word", Karma Soft Systems, USA, 2011

[7]. Llia Smirnov, "Overview of Stemming Algorithms", DePaul University, 2008

[8]. Willett Peter, "The Porter stemming algorithm: then and now", White Rose University Consortium, 40 (3). pp. 219223, 2006

[9]. Steven Abney, "Parts-Of -Speech Tagging and Partial Parsing", Columbia University, Computer Science Department, 1996

[10]. www.nlp.stanford.edu, Grammar check and spell check 